\title{
Creation of Dark Solitons and Vortices in Bose-Einstein Condensates
}

\author{
R. Dum ${ }^{1,2,3}$, J.I. Cirac ${ }^{1,2}$, M. Lewenstein ${ }^{4}$, and P. Zoller ${ }^{1}$, \\ ${ }^{1}$ Institute für Theoretische Physik, Universität Innsbruck, \\ A-6020 Innsbruck, Austria \\ ${ }^{2}$ Departamento de F'rsica Aplicada, Universidad de Castilla-La Mancha, 13071 Ciudad Real, Spain \\ ${ }^{3}$ Ecole Normale Supérieure, Laboratoire Kastler Brossel, \\ 24, Rue Lhomond, F-75231 Paris Cedex 05, France \\ 4 Commissariat à l'Energie Atomique, DSM/DRECAM/SPAM, Centre d'Etudes de Saclay \\ 91191 Gif-sur-Yvette, France
}

(September 20, 2018)

We propose and analyze a scheme to create dark solitons and vortices in Bose-Einstein condensates. This is achieved starting from a condensate in the internal state $|a\rangle$ and transferring the atoms to the internal state $|b\rangle$ via a Raman transition induced by laser light. By scanning adiabatically the Raman detuning, dark solitons and vortices are created.

03.75.Fi,05.30.Jp

Recently Bose-Einstein condensation has been demonstrated in dilute atomic gases [1]. This state of matter resembles other states found in the fields of superfluidity, superconductivity and nonlinear optics. It is thus natural to expect that some phenomena that appear in those fields, such as solitons and vortices, can be observed with dilute atomic gases. In fact, the Gross-Pitaevskii Equation (GPE) 2] which describes the wavefunction of the macroscopically occupied state in a trapping potential allows for stationary solutions that represent dark solitons (see below) and vortices (see also [3]). Here we propose a scheme to generate these solutions in a controlled way using an approach based on "engineering the macroscopic wavefunction": once the condensate has formed, we use a coherent Raman process to create solitons and vortices.

Our idea is to couple the internal state $|a\rangle$ where the condensate is formed with another internal state $|b\rangle$ using a Raman transition (Fig. 1). The laser parameters are chosen such that the state after the transfer is an eigenstate of the GPE corresponding to solitons or vortices. The complete transfer is achieved by an adiabatic change of the laser frequency. In the present case, where the whole process is described by non-linear equations, the familiar idea of adiabatic transfer along eigenstates has to be taken with caution since the Hamiltonian describes the atomic interactions by a mean field depending on the shape of the wavefunctions. We will analyze two cases: in 1D we will study the creation of dark solitons and in $2 \mathrm{D}$ the creation of vortices.

In the Hartree-Fock approximation the state $\Phi$ of a condensate of $N$ bosons confined in a potential $V(\vec{r})$ is described by the time-independent GPE

$$
\left[-\frac{\hbar^{2} \vec{\nabla}^{2}}{2 m}+V(\vec{r})+N g|\Phi(\vec{r}, t)|^{2}\right] \Phi(\vec{r}, t)=E \Phi(\vec{r}, t) .
$$

The mean field interactions are characterized by a coupling constant $g=4 \pi \hbar^{2} a_{s} / m$, where $a_{s}>0$ is the $s$-wave scattering length. In the following analysis we will concentrate on the Thomas-Fermi limit [4], since most of the experiments operate in this regime [i]. In this limit, the mean interaction energy is much larger than the mean kinetic energy which can be neglected when calculating the ground state solution of the GPE (1):

$$
\Phi_{E_{0}}(\vec{r})=\left\{\left[E_{0}-V(\vec{r})\right] /(N g)\right\}^{1 / 2} .
$$

for $\vec{r}$ such that $V(\vec{r})<E_{0} ; E_{0}$ is determined from $\int d^{3} r\left|\Phi_{E_{0}}(\vec{r})\right|^{2}=1$ which reflects particle conservation. Here we are interested in other stationary solutions of (11) $\Phi(\vec{r})$ with energy larger than $E_{0}$. Far from the trap center $\vec{r}=0$ we can still neglect the kinetic energy which suggests the ansatz

$$
\Phi(\vec{r})=\phi(\vec{r}) \Phi_{E}(\vec{r})
$$

that is a a product of an envelope function (2) and a function $\phi$ with the condition $|\phi(\vec{r})| \simeq 1$ far away from the origin. Now $E$ is determined from $\int d^{3} r\left|\phi(\vec{r}) \Phi_{E}(\vec{r})\right|^{2}=1$. Substituting (3) in the GPE and neglecting the derivatives of $\Phi_{E}(\vec{r})$ we obtain a nonlinear equation for $\phi(\vec{r})$, which near $\vec{r}=0$, where we can neglect the variation of the trapping potential, reads

$$
\left[-\frac{\hbar^{2} \vec{\nabla}^{2}}{2 m}+N g|\phi(\vec{r})|^{2}\right] \phi(\vec{r})=E \phi(\vec{r}) .
$$

This equation has the same form as the familiar GPE for the homogeneous case. Together with the boundary conditions $|\phi(\vec{r})| \simeq 1$ for $|\vec{r}| \rightarrow \infty$ it gives rise to dark solitons and vortices [5]. Our goal is to design bosonlaser interactions which will generate these solutions.

We assume that the bosons have two internal levels $|a\rangle$ and $|b\rangle$ as in $\mathrm{Rb}[$ [6]. The particles interact with a laser beam that connects these two levels. The evolution of 
the mean field spinor $\vec{\Phi}=\left(\Phi_{a}, \Phi_{b}\right)$ obeys the following non-linear equation

$$
i \hbar \frac{d}{d t} \vec{\Phi}(\vec{r}, t)=\left(\mathcal{H}+\mathcal{H}_{1} \vec{\Phi}(\vec{r}, t)\right.
$$

where

$$
\mathcal{H}=-\frac{\hbar^{2} \vec{\nabla}^{2}}{2 m}+V(\vec{r})+N g\left[\left|\Phi_{a}(\vec{r}, t)\right|^{2}+\left|\Phi_{b}(\vec{r}, t)\right|^{2}\right],
$$

describes the linear evolution plus atom-atom interactions and

$$
\mathcal{H}_{1}=\left(\begin{array}{cc}
0 & \frac{1}{2} \lambda(\vec{r}) \\
\frac{1}{2} \lambda(\vec{r}) & -\delta
\end{array}\right)
$$

the interaction with the laser. In (6) we have assumed that the interaction between levels $i$ and $j(i, j=a, b)$ can be described by a pseudopotential $g_{i j} \delta(\vec{r})$ and that $g_{i j}=g>0$, which indeed is a good approximation for Rubidium [6]. For $V(\vec{r})$ we choose an anisotropic harmonic potential with frequencies $\omega_{x, y, z}$ which we assume identical for both internal levels. In (7),$\lambda(\vec{r})=$ $\Omega_{a}(\vec{r}) \Omega_{b}(\vec{r}) /(4 \Delta)$ where $\Delta$ is the detuning from the intermediate level $|r\rangle$, and $\Omega_{a, b}$ are the Rabi frequencies corresponding to the couplings between $|a\rangle$ and $|b\rangle$ to $|r\rangle$, respectively (see Fig. 1). Their specific form depends on the laser configuration. The Raman twophoton detuning is denoted by $\delta$. The conservation of the number of particles gives the normalization condition $\int d^{3} \vec{r}\left[\left|\Phi_{a}(\vec{r})\right|^{2}+\left|\Phi_{b}(\vec{r})\right|^{2}\right] \equiv \pi_{a}^{2}+\pi_{b}^{2}=1$ with $\pi_{a, b}^{2}$ the populations in levels $a, b$, respectively.

As the initial state we take $\Phi_{E_{0}}$ [see Eq.(2)] which corresponds to the state of the condensate formed in the internal level $|a\rangle$. We will design $\lambda(\vec{r})$ and $\delta$ such that the atoms are transferred to $|b\rangle$ with a wavefunction which corresponds to dark solitons or vortices. In absence of interactions $(g=0)$ the problem reduces to the one of a single trapped particle. In that case, one can simply use a resonant $\delta=0$ laser pulse of a well defined area to carry out the population transfer [7]. In presence of interactions, this method will not work: as soon as particles are transferred to a different state and the shape of the wavefunction changes, the interaction energy changes [Fig. 1(a,b)]. Therefore, an initially resonant Raman laser pulse at the beginning of the pulse soon becomes off-resonant, and the transfer process will stop. We circumvent this problem by using adiabatic passage. The idea is to start from a negative Raman detuning so that the atoms do not feel the laser [Fig. 1(a)]. Then, the Raman detuning is changed adiabatically to sufficiently large positive values [Fig. 1(b)]. As soon as the laser frequency approaches the Raman resonance, the atoms will start flowing to the state $|b\rangle$. The fact that the interaction energy changes will effectively change the value of $\delta$ at which this resonance occurs. This will not affect the overall process provided the final value of $\delta$ is large enough so that at the end the atoms do not feel the off-resonant laser anymore. The reason is that adiabatic transfer only depends on the initial and final values of the adiabatic parameter (detuning).

In order to describe analytically the adiabatic process we look for stationary solutions $\vec{\Phi}_{\delta}(\vec{r})$ of (5) for a given value of $\delta$. The idea is to change $\delta$ adiabatically so that the state of the system changes according to $\vec{\Phi}_{\delta}$ in accordance to the adiabatic theorem. We thus have to impose that for the initial and the final Raman detunings $\delta_{0}$ and $\delta_{f}$ the spinor $\vec{\Phi}_{\delta}$ corresponds to the initial state and the desired state, respectively. That is: (i) for $\delta \rightarrow \delta_{0}$, $\vec{\Phi}_{\delta} \simeq\left(\Phi_{E_{0}}, 0\right)$; (ii) for $\delta \rightarrow \delta_{f}, \vec{\Phi}_{\delta}=\left(0, \Phi_{b}\right)$ where $\Phi_{b}$ is another stationary solution of Eq. (1). In order to find the appropriate stationary solutions of (5) we have to solve first the set of nonlinear equations

$$
\mathcal{H} \Phi_{a, b}^{\pi_{a}}(\vec{r})=\epsilon_{a, b}^{\pi_{a}} \Phi_{a, b}^{\pi_{a}}(\vec{r}),
$$

for a fixed (real) value of $\pi_{a}$ (with $\pi_{b}=\left|1-\pi_{a}^{2}\right|^{1 / 2}$ due to normalization) and satisfying the conditions (i,ii). Here $\Phi_{a, b}^{\pi_{a}}$ describe the wavefunctions in absence of laser coupling for given populations $\pi_{a, b}$. The presence of the laser will lead to a dressing of these levels in analogy with the well-known picture known from non-interacting atoms in presence of laser light [8]. Once these functions are found, we replace $\mathcal{H} \vec{\Phi}$ in (5) by (8), that is we restrict the evolution to the subspace defined by these two functions; by multiplying the first equation by $\Phi_{a}(\vec{r})^{*}$ and the second by $\Phi_{b}(\vec{r})^{*}$ and integrating we obtain

$$
\left(\begin{array}{cc}
\epsilon_{a}^{\pi_{a}} & \lambda^{\pi_{a}} \\
\lambda^{\pi_{a} *} & \epsilon_{b}^{\pi_{a}}-\delta
\end{array}\right)\left(\begin{array}{c}
\pi_{a} \\
\pi_{b}
\end{array}\right)=E\left(\begin{array}{c}
\pi_{a} \\
\pi_{b}
\end{array}\right),
$$

where we have defined

$$
\lambda^{\pi_{a}}=\frac{1}{2 \pi_{a} \pi_{b}} \int d^{3} \vec{r} \lambda(\vec{r}) \Phi_{a}^{\pi_{a}}(\vec{r})^{*} \Phi_{b}^{\pi_{a}}(\vec{r}) .
$$

Equations (9) define two generalized dressed states of our system which take fully into account atomic interactions. From these equations we can determine the values of $\delta$ and $E$ corresponding to $\Phi_{a, b}^{\pi_{a}}$. In summary, the problem is reduced to solve the coupled eigenvalue equations (8) for a given value of $\pi_{a}$, such that varying continuously this parameter we go from $\Phi_{a}(\vec{r})=\Phi_{E_{0}}(\vec{r})$ to the desired state $\Phi_{b}(\vec{r})$. The energy separation $\Delta_{a, b}\left(\pi_{a}\right) \equiv \epsilon_{b}^{\pi_{a}}-\epsilon_{a}^{\pi_{a}}$ between the bare wavefunctions $\Phi_{a, b}^{\pi_{a}}$ gives the resonance condition for the Raman detuning, $\delta \rightarrow \Delta_{a, b}\left(\pi_{a}\right)$, for fixed level populations $\pi_{a, b}$. The induced width is given by $\lambda\left(\pi_{a}\right)$ for $\pi_{a}^{2} \simeq 1 / 2$ which gives the avoided crossing and therefore the time scale for adiabaticity.

We illustrate this procedure now for the $1 \mathrm{D}$ case. This corresponds to the limit in which $\omega_{x, y} \gg \omega_{z}$ so that the dynamics along the $x$ and $y$ direction is frozen. Our goal is to create a dark soliton starting from $\Phi_{E_{0}}(z)$. We are interested in dark solitons with a zero at the trap center. 
This requires that the laser interaction changes the parity of the wavefunction when the atoms are transferred from a to b. To this aim we choose the simplest laser configuration, so that $\lambda(z)=\lambda_{0} \sin (k z)$, i.e. a standing wave. In order to achieve an efficient coupling we take $k \leq 1 / z_{0}$, where $z_{0}$ is the size of the $\Phi_{E_{0}}[9]$; note that the effective $\lambda$ defined in (10) will be very small if $k z_{0} \gg 1$. In this case the avoided crossing of the dressed energy levels will be of the order $\lambda_{0}$, which sets the time scale for the adiabaticity. On the other hand, $\lambda_{0}$ has to be smaller than the typical energy separations $\left|\Delta_{a b}\right|$ so that the Stark shifts do not mix these wavefunctions with others of higher energies $\epsilon$. The initial value of the Raman detuning must be $\delta_{0} \ll \Delta_{a, b}\left(\pi_{a}=1\right)$, whereas the final value must fulfill $\delta_{f} \gg \Delta_{a, b}\left(\pi_{a}=0\right)$. In Fig. 2 we have plotted numerical results of the solutions of the time-dependent GPE (5). Figure 2(a) shows the spatial distribution $P_{a, b}(z)=\left|\Phi_{a, b}(z)\right|^{2}$ corresponding to states $|a\rangle$ (solid line) and $|b\rangle$ (dashed line). As the transfer progresses, we see that the wavefunction of the atoms in $|a\rangle$ narrows and the one of the atoms in $|b\rangle$ develops a hole in the center. When most of the atoms are in b, the corresponding wavefunction contains a dark soliton. This manifests itself in the effective (trap plus mean field) potential; it is initially flat, and later it develops a narrow dip as a consequence of the dark soliton [see Fig. 1(b)]. The part of the atoms still in $|a\rangle$ become trapped in a bound state of this dip, which becomes deeper as we move more atoms in the excited state. In Fig. 2(b) we have plotted the fraction of atoms $P_{a, b}=\pi_{a, b}^{2}$ of levels a and b. As this figure shows, the transfer efficiency is essentially 100 percent. For the analytical understanding of these results, we proceed as explained above in terms of generalized dressed states. First, in the limit $\pi_{a} \rightarrow 1$ one can estimate the value of $\Delta_{a b}$ using a square well of length equal to the size of the Thomas Fermi solution $\Phi_{E_{0}}$. In the opposite limit, $\pi_{a} \rightarrow 0$ we can calculate $\Delta_{a b}$ in the same way as in the context of Eq. (2). We write $\vec{\Phi}(z)=\left[\phi_{a}(z), \phi_{b}(z)\right] \Phi_{E}(z)$, with $\Phi_{E}(z)$ defined in (2). Near the trap center we obtain

$$
\left[-\frac{\hbar^{2}}{2 m} \frac{d^{2}}{d z^{2}}+N g\left|\phi_{a}(z)\right|^{2}+\epsilon_{b}\left|\phi_{b}(z)\right|^{2}\right] \phi_{a, b}(z)=\epsilon_{a, b} \phi_{a, b}(z),
$$

with the boundary conditions $\left|\phi_{a}(z)\right| \rightarrow 0$ and $\left|\phi_{b}(z)\right| \rightarrow$ 1 as $|z| \rightarrow \infty$. We find that $\phi_{a}(z)=A \operatorname{sech}(\alpha z)$ and $\phi_{b}(z)=\tanh (\alpha z)$ solve Eq. (11). With the normalization condition we can find the values of $A, \alpha$ and $\epsilon_{a, b}$ for a given value of $\pi_{a}$. This solution is in perfect agreement with our 1D numerical results. We performed a full $3 D$ integration of the GPE in order to make sure that the $1 D$ effect is not effected by the presence of the transverse degrees of freedom [10].

A two-soliton solution is obtained by starting from the ground state in a, and coupling with a laser configuration which preserves the parity, $\lambda(z)=\lambda_{0} \cos (k z)$. In order not to couple to the ground state in $b$, the initial detuning has to be $\delta_{0}>\left|\lambda_{0}\right|$. We then increase the detuning adiabatically to a sufficiently large value. In Fig. 3 we show plots of numerical solutions. At the end of the process all the particles are in the state $|b\rangle$ with a wavefunction that includes two dark solitons. Again an analytical Ansatz is possible: for $\left|\pi_{b}\right| \rightarrow 1$ we set $\Phi_{b}(z)=\phi_{b}(z) \Phi_{E}(z)$ with $\phi_{b}(z)=\tanh [\alpha(z-a)] \tanh [\alpha(z+a)]$ ( $a$ is a free parameter) which reproduces the numerical results very well.

A 2D situation arises in the limit $\omega_{z} \gg \omega_{\perp}=\omega_{x}=$ $\omega_{y}$. In this limit we are interested in creating vortex solutions of the form $\Phi(\rho, \varphi)=f(\rho) e^{i \varphi}$ where $\rho$ and $\varphi$ are cylindrical coordinates and $f(\rho)$ is a function with a zero at $\rho=0$. In order to provide the required angular momentum to the atoms that are transferred, we choose a laser configuration such that $\lambda(x, y)=\lambda_{0}\left[\sin \left(k_{L} x\right)+\right.$ $\left.i \sin \left(k_{L} y\right)\right] \simeq \lambda_{0} k_{L} \rho e^{i \varphi}$ for $k_{L} \rho \lesssim 1$ [11]. The density distribution $|\Phi(x, y)|^{2}$ after an adiabatic switch of the detuning is plotted in Fig. 4. The insert shows that all the population is transferred to the vortex state. Analytical approximations can be obtained with the ansatz $\phi_{a}(\vec{r})=$ $A \operatorname{sech}(\alpha \rho)$ and $\phi_{b}(\vec{r})=\tanh (\alpha \rho) e^{i \varphi}$.

A simple way of observing the shape of the density $n(\vec{r})$ is by opening of the trap. In a way similar to Ref. [12] we can show that the density at later times is related to the density at $t=0$ by $n(\vec{r}, t)=n[\vec{r} / \gamma(t), 0] / \gamma^{2 d}(t)$ where the scaling factors obey $\ddot{\lambda}=\omega^{2} / \lambda^{d+1}$ ( $d$ is the dimension) and initial conditions $\lambda=1, \dot{\lambda}=0$. This leads to an asymptotic behavior $\gamma(t) \rightarrow \sqrt{2} \omega_{z} t$ for the 1D dark soliton and $\gamma(t) \rightarrow \omega_{\perp} t$ for the vortex solution. This selfsimilar expansion without a change in shape is typical for solitonic behavior. On the other hand, another important issue to address is the stability of vortices and dark solitons. In some recent works dealing with excitations of vortex states [3] it is shown that quasiparticles states localized near the center of the trap will be preferentially occupied by collisions thereby destabilizing the vortex. However, using the analogy between our proposal and the excitations of condensates via time-dependent trapping potentials [13], we expect the destabilization time to be much longer than that required for the creation of vortices and solitons. The reason is that the creation of vortices and dark solitons involve regular (non-chaotic) solutions of the GPE, which give rise to destabilizations that grow only polynomially with time (instead of exponentially) [13].

We have demonstrated that one can engineer the macroscopic wavefunction of Bose-Einstein condensed sample by coupling the internal atomic levels with a laser. The method is based on adiabatic transfer of population along generalized dressed states which include the nonlinear atom-atom interactions. We find the technique to be very robust against uncertainties in the parameters of the problem. Furthermore, we have developed analytical approximations to describe this process, and show with 
explicit examples how to generate dark solitons and vortices. Our numerical results confirm our predictions and demonstrate the stability of these solutions. We expect that this method can be applied to current or planned experiments.

We thank K. Burnett for discussions. This work was supported in part by the TMR network ERB-FMRXCT96-0087, and by the Austrian Science Foundation.

[1] M. H. Anderson et al., Science 269, 198 (1995); K. Davis et al., Phys. Rev. Lett. 75, 3969 (1995); C.C. Bradley et al., Phys. Rev. Lett. 78, 985 (1997).

[2] M. Edwards et al., Phys. Rev. A 53, R1950 (1996); F. Dalfovo, S. Stringari, Phys. Rev A 53, 2477 (1996).

[3] R.J. Dodd et al., Phys. Rev A 56, 587 (1997); D.S. Rokhsar, Phys. Rev. Lett. 79, 2164 (1997).

[4] G. Baym and C. Pethick, Phys. Rev. Lett. 76, 6 (1996).

[5] V.E. Zakharov, A.B. Shabat, JETP 37, 823 (1973);

[6] C.J. Myatt et al.,Phys. Rev. Lett. 78, 586 (1997).

[7] See, e.g., J. I. Cirac et al., Adv. At. Mol. Phys. 37, 237 (1996).

[8] C. Cohen-Tannoudji et al., Photon-Atom Interactions (Wiley 1992).

[9] For typical experimental conditions a typical wavelength is $500 \mathrm{~nm}$ and $z_{0}$ is $\approx 5 \mu \mathrm{m}$; therefore one has to use two laser beams forming a small angle to achieve $k_{L} z_{0} \simeq 1$.

[10] We take $\omega_{z} / \omega_{\perp}=0.1$ and we relate the interactions by $g_{1 D}=\frac{1}{\pi} \frac{g_{3 D}}{a_{\perp}^{2}}$ where $a_{\perp}=\left(\hbar / m \omega_{\perp}\right)^{1 / 2}$.

[11] A coupling of this form can be achieved using Raman beams with wave vectors $\vec{k}_{1,2}$ such that $\left|\vec{k}_{1}-\vec{k}_{2}\right|<k_{L}$. The cross terms with wavenumber $\overrightarrow{k_{1}}+\overrightarrow{k_{2}}$ can be neglected due to Lamb-Dicke suppression.

[12] Y. Castin and R. Dum, Phys. Rev. Lett. 77, 5315 (1996).

[13] Y. Castin and R. Dum, Phys. Rev. Lett. 79, XXXX (1997).

FIG. 1. Schematic representation of the process: (a) initial state; (b) final state.

FIG. 2. Generation of a dark soliton. The detuning is varied linearly with time from $\delta=-1.5 \omega$ to $\delta=6.5 \omega$. Other parameters: $\lambda_{0}=0.15 \omega, k_{L}=0.5 / a_{0}$ and $N g=50 \hbar \omega a_{0}$, where $a_{0}^{2}=\hbar /(m \omega)$. (a) snap shots of the position distributions of the wavefunctions corresponding to atoms in level $|a\rangle$ (solid line) and $|b\rangle$ (dashed line) for different times; (b) Populations of these levels as a function of time.
FIG. 3. Generation of two dark solitons. The detuning is varied linearly with time from $\delta=0.25 \omega$ to $\delta=5 \omega$. Other parameters: $\lambda_{0}=0.15 \omega, k_{L}=0.5 / a_{0}$ and $N g=20 \hbar \omega a_{0}$, where $a_{0}^{2}=\hbar /(m \omega)$.

FIG. 4. Generation of a vortex: position distribution of the final state. The detuning is varied linearly from $\delta=-0.6 \omega_{\perp}$ to $\delta=5 \omega_{\perp}$. The parameters are $N g=500 \hbar \omega_{\perp} a_{\perp}^{2}, \lambda=0.15 \hbar \omega_{\perp}$ and $k_{L}=0.5 a_{\perp}$. The inset shows the evolution of the populations in levels $|a\rangle$ and $|b\rangle$ (solid and dashed lines, respectively). 

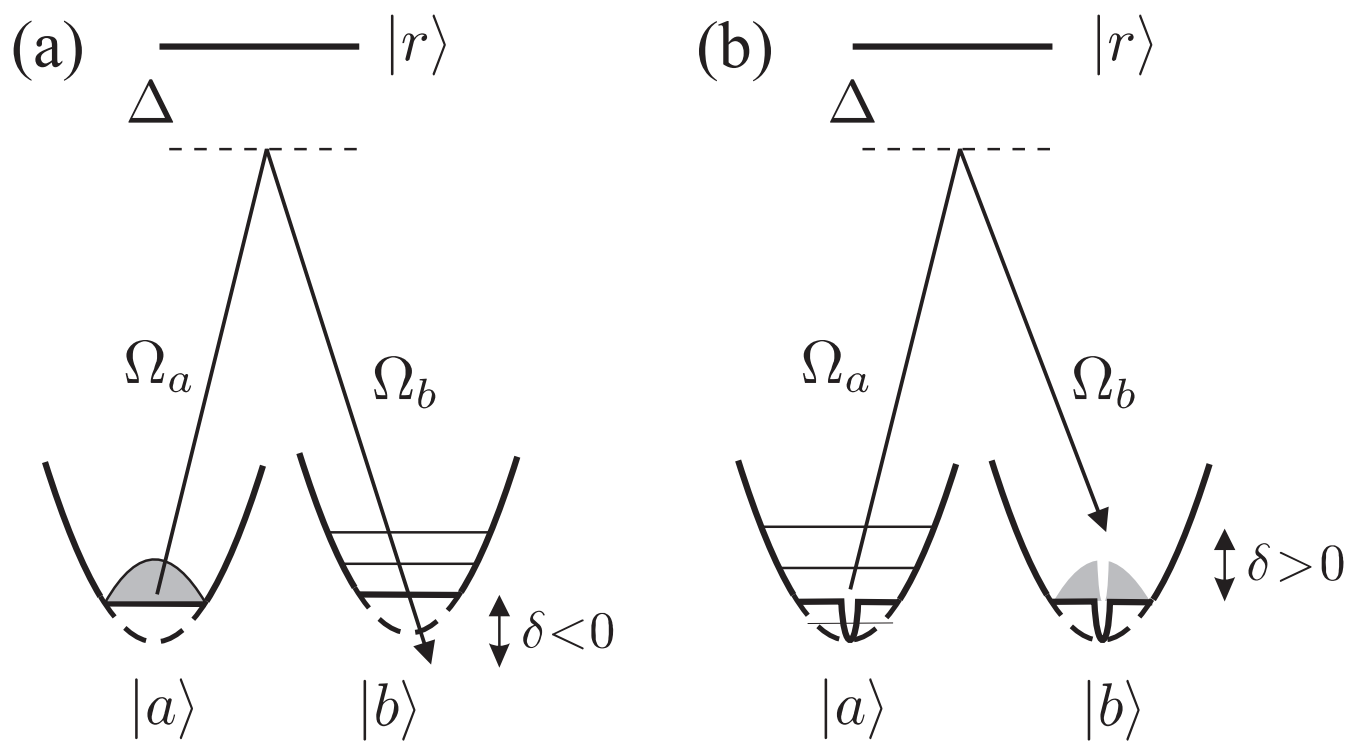

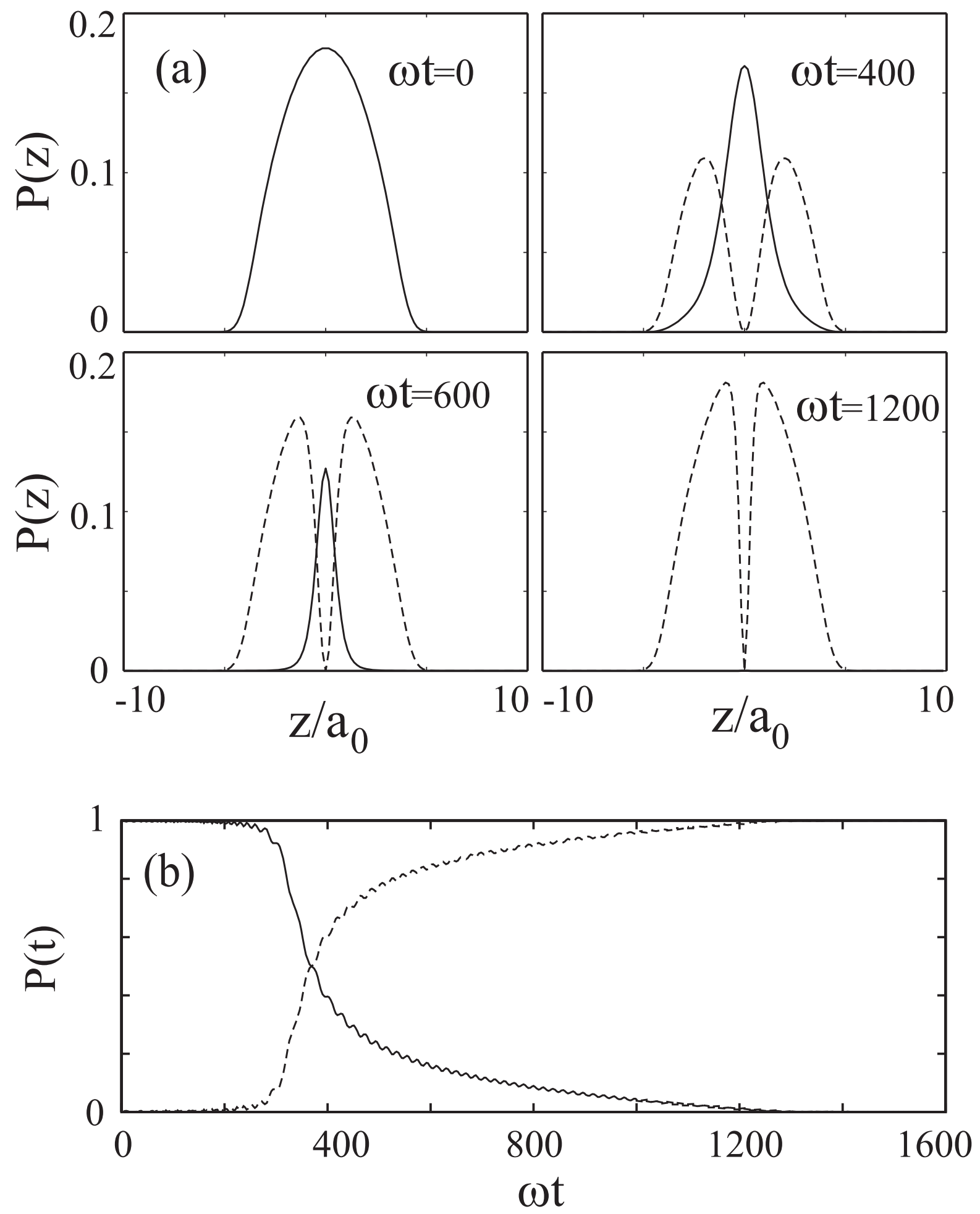

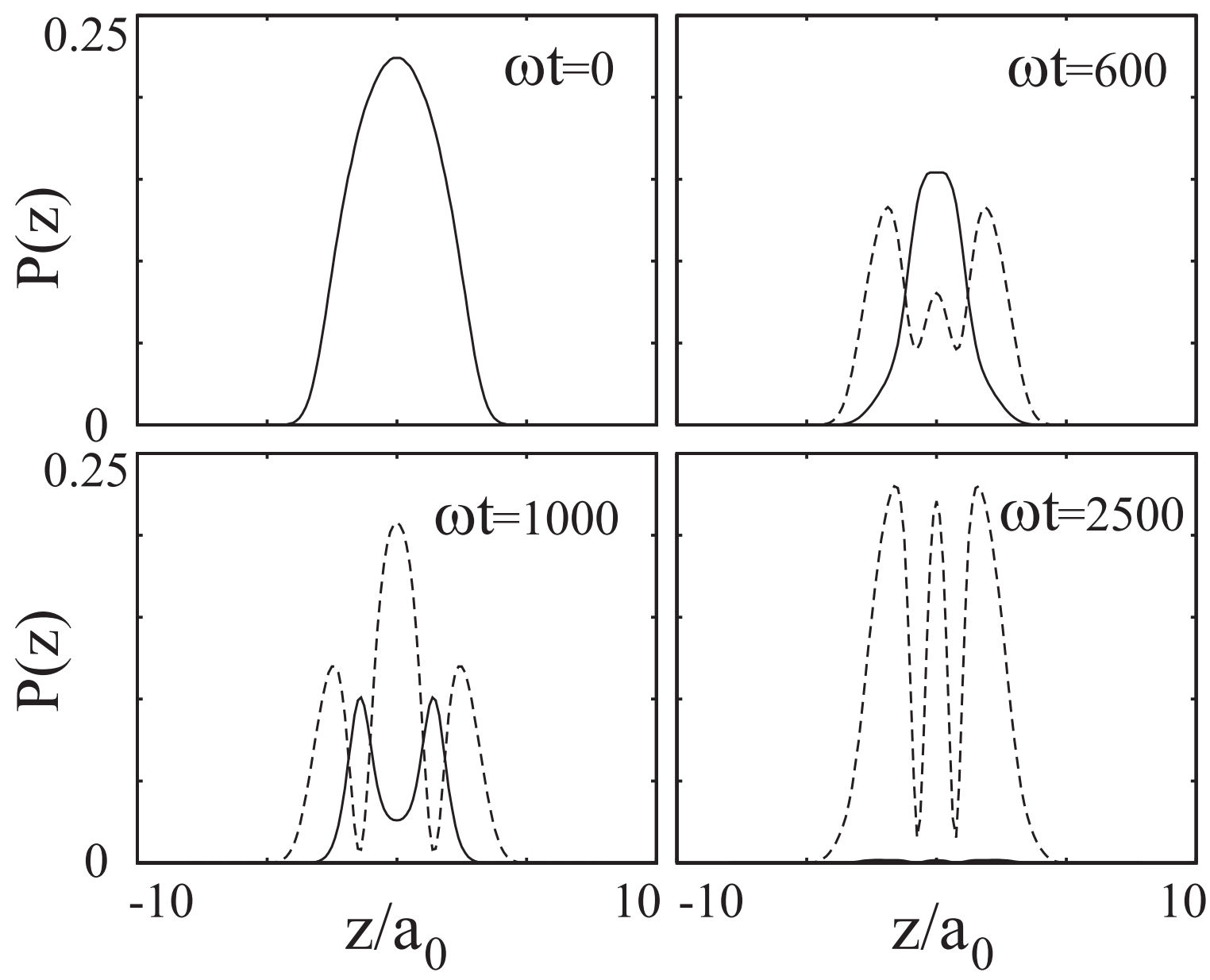


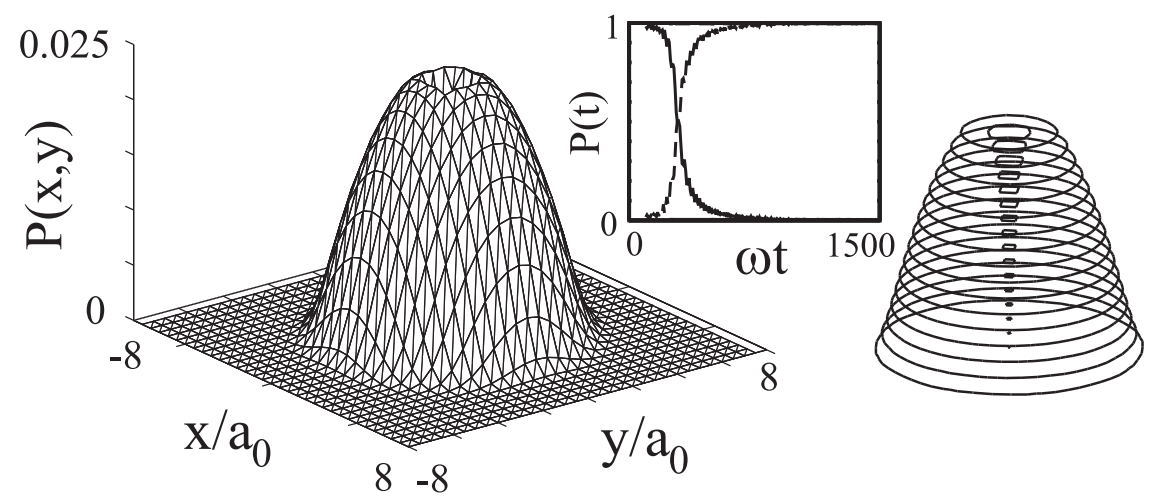

